\title{
Change of the initiation time of blood coagulation in pregnancy from 10-months to postpartum
}

\author{
Toshiaki Sagesaka ${ }^{\mathrm{a}, *}$ and Hiroyuki Funabashi ${ }^{\mathrm{b}}$ \\ ${ }^{a}$ Department of Obstetrics and Gynecology, International University of Health and Welfare, \\ Atami Hospital, Atami-shi, Shizuoka, Japan \\ ${ }^{\mathrm{b}}$ Funabashi Ladies Clinic, Morokawa, Koga-shi, Ibaraki, Japan
}

\begin{abstract}
We measured the time of initiation of blood coagulation (Ti) from pregnancy 10-months (36 40 weeks) till 1-month after delivery, paying particular attention to the very early postpartum period, using a damped oscillation rheometer that is approximately 160 times more sensitive than the Thromboelastogram ${ }^{\circledR}$ to evaluate the risk of thrombus formation. Blood samples were obtained from healthy volunteers at pregnancy 10-month, 1-hour, 3-hours, 4-days, 7-days, 3-weeks and 1-month after delivery. Ti values at pregnancy 10-month, 1-hour, 3-hours, 4-days, 7-days, 3-weeks, 1-month after delivery and in non-pregnant females were $20.4 \pm 2.2,11.7 \pm 1.6,13.2 \pm 3.1,17.2 \pm 2.0,20.2 \pm 1.6,21.4 \pm 4.0,24.6 \pm 3.6$, and $(25.0 \pm 3.4)$ minutes, respectively. Ti was significantly shorter at pregnancy 10-month, 1-hour, 3-ours, 4-days, 7-days and 3-weeks after delivery than in non-pregnant females. These data show that the blood of pregnant females is more hypercoagulable than nonpregnant females from pregnancy 10-month until 3-weeks post delivery, suggesting that they are at high risk of VTE after discharge from hospital.
\end{abstract}

Keywords: Blood coagulation, postpartum, thrombus formation, damped oscillation rheometer

\section{Introduction}

Pregnancy and the postpartum period are associated with a four-and 14-fold increased risk of deep vein thrombosis (DVT), respectively [22]. The frequency of thrombosis is similar throughout the three trimesters [8] and thrombotic risk is highest after caesarean section [9]. The intrinsic blood coagulation pathway appears to be activated during pregnancy [13]. A decrease in the elevated level of factor XII occurs at delivery and factor XI decreases below normal during late pregnancy. During delivery, the vasculature in the placenta bed degenerates; thus, collagen may be exposed and may activate the intrinsic system [13]. Gerbasi et al. [7] reported that the accelerated state of hemostatic activity that appears to peak immediately after placental delivery and for the first three hours post partum, as measured by determination of fibrinopeptide A, B-thromboglobulin and platelet factor 4, as indicators of coagulation activity. The increase in activity was followed by a gradual decrease during the first 48 hours post partum.

\footnotetext{
${ }^{*}$ Corresponding author: Toshiaki Sagesaka, Department of Obstetrics and Gynecology, International University of Health and Welfare, Atami Hospital, 13-1, Higashikaigan-cho, Atami-shi, 413-0012 Shizuoka, Japan. Tel.: 81557 81 9171; ext 3610; Fax: +81 55783 6632; Home Phone and Fax: +81 33813 6484; E-mail: QYT07667@ nifty.ne.jp.
} 
These data suggest that the blood coagulation activity may increase immediately after delivery compared with in pregnancy 10 -months $(10 \mathrm{M})$.

In gynecologic area, von Tempelhoff et al. [28] reported a big prospective study about blood fluidity, coagulation and thrombosis in patients with gynecologic malignancy. However, very few studies have systematically examined the longitudinal changes in coagulation that occur during labor, after delivery and during the very early postpartum period [5]. Global tests, such as Thromboelastogram ${ }^{\circledR}$ (TEG) or Sonoclot signature, etc., that measure hemostasis in whole blood or citrated blood, with or without activators, appear to closely resemble the in vivo situation, although they do not reflect the interaction(s) between the blood and endothelium [12]. We measured the initiation time of blood coagulation (Ti) from pregnancy $10 \mathrm{M}$ till one month after delivery, focusing particularly on the very early postpartum period, using a damped oscillation rheometer that is approximately 160 times more sensitive than that of TEG, to evaluate the risk of thrombus formation [17].

\section{Materials and methods}

The study was approved by the International University of Health and Welfare Human Biology Research Ethics Committee. The manuscript was written according to the guidelines of the journal [1].

\subsection{Rheometer}

The coagulation of blood samples that had been placed in polypropylene tubes was measured using a damped oscillation rheometer. This rheometer in Fig. 1A is described in detail elsewhere [15, 16, 18]. The logarithmic value of the damping factor (LDF) is closely related to fluidity, that is, the viscosity and/or viscoelasticity of the blood during progression of the coagulation process.

\subsection{Blood samples}

Blood samples were obtained from healthy volunteers at pregnancy $10 \mathrm{M}$ (36 40 weeks, $28.4 \pm 4.2$ years old, mean $\pm \mathrm{SD}$ ), 1-hour, 3-hours, 4-days, 7-days, 3-weeks and 1-month (28 33 days: mean 31.1 days) after delivery. Fifteen samples were collected at each time-point. They were dealt according to the guidelines [10] of measurement of blood viscosity and erythrocyte deformability. Blood was collected from a peripheral vein via a 21-gauge needle using a two-syringe technique. The first sample was discarded to avoid tissue contamination of the blood, and the second sample was used for the rheometer measurements and other laboratory tests. Immediately after collection, 1/10 volume of $3.2 \%$ trisodium citrate dihydrate was added to the blood as an anticoagulant. The blood samples were also examined for APTT, PT, and fibrinogen concentrations.

\subsection{Measurement of the change of viscosity}

Measurements were performed within 3 hours after blood collection according to the guidelines [4]. Fig. $1 \mathrm{~B}$ shows a typical rheometer result. After incubation at $37^{\circ} \mathrm{C}$ for 5 minutes, $85 \mu \mathrm{l}$ of $0.25 \% \mathrm{CaCl}_{2}$ was added to each 1-ml samples in polypropylene tubes. After this $\mathrm{CaCl}_{2}$ addition, defined as zero time, the sample was immediately poured into the tube, and the LDF during coagulation was measured. The Ti was 


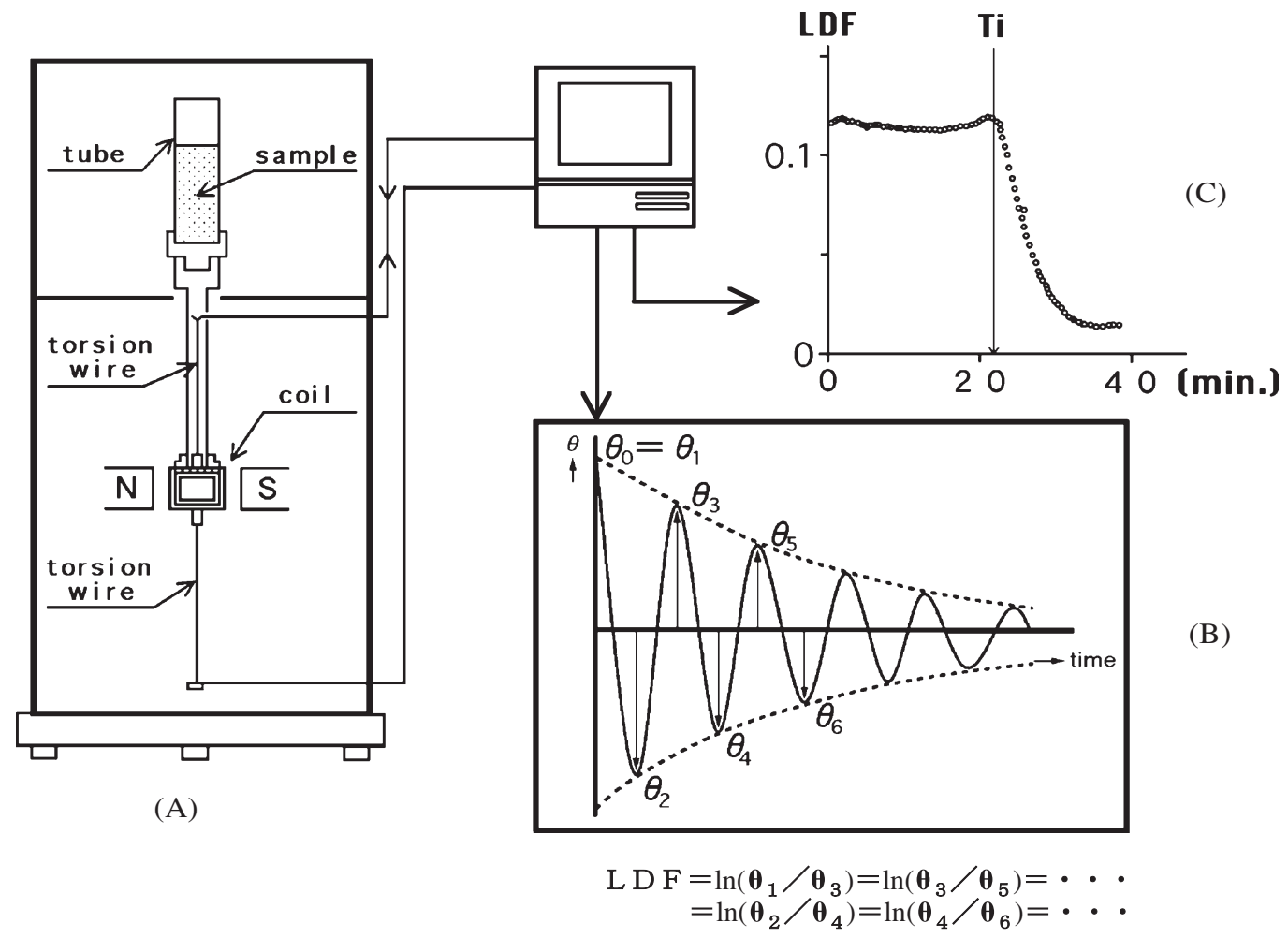

Fig. 1. A damped oscillation rheometer and the related graphs. LDF: logarithmic damping factor, Ti: the time of initiation of coagulation. (A) A schematic diagram of damped oscillation rheometer. (B) A damped oscillation curve (C) Schematic results obtained with the rheometer.

determined from the intersection of the maximum value of the LDF and the maximum slope of the LDF, as shown by the arrow in the LDF curve in Fig. 1C. The Ti represents the onset of fibrin polymerization, which was determined by computational analysis. Changes in the structure of fibrin polymerization during coagulation were examined to confirm the Ti, using a scanning electron microscope [14].

\subsection{Statistic treatment}

All statistical tests were conducted using the unpaired $t$-test (2-tail). $P$-values $<0.05$ were accepted as statistically significant.

\section{Results}

1) APTT, PT, and fibrinogen concentrations

The original bloods from the volunteers were examined for APTT, PT, and fibrinogen concentrations, all of which were within the normal ranges. 


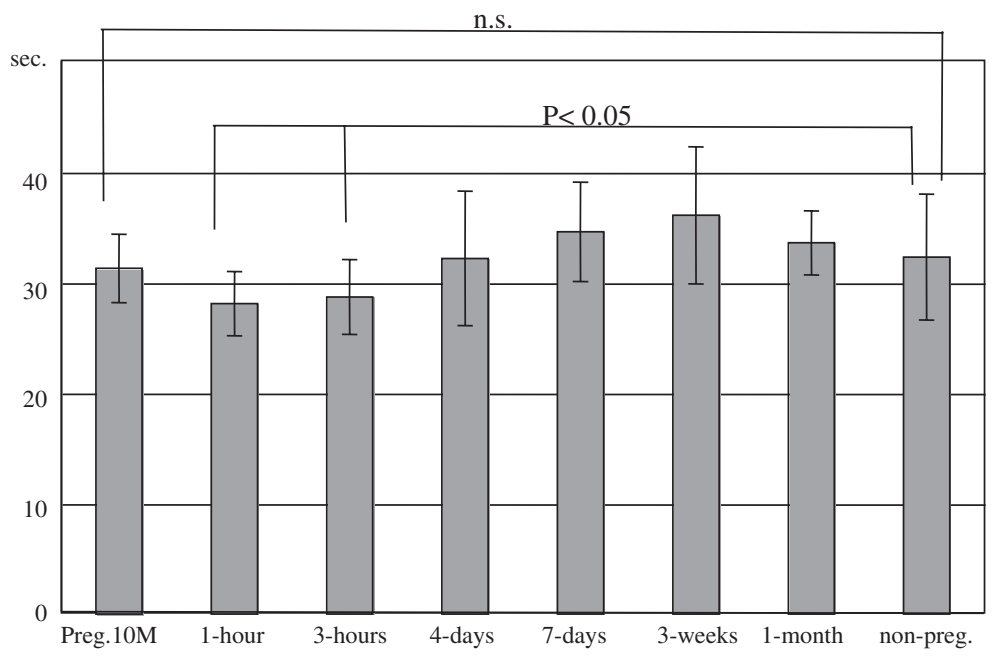

Fig. 2. APTT change. No significant differences between the adjoining groups with the level of significance of $5 \%$.

\section{2) Blood count}

CBCs were determined in all samples and they were within normal platelet concentrations.

\section{3) Change in APTT (Fig. 2)}

The APTT values in pregnancy $10 \mathrm{M}, 1$-hour, 3-hours, 4-days, 7-days, 3-weeks, 1-month after delivery and in non-pregnant females were $31.3 \pm 3.1,28.1 \pm 2.9,28.7 \pm 3.4,32.2 \pm 6.1,34.6 \pm 4.5,36.1 \pm 6.2$, $33.6 \pm 2.9$, and $(32.3 \pm 5.7)$ seconds, respectively. APTT at pregnancy $10 \mathrm{M}$ showed no significant difference from that during non-pregnancy. However, APTT at 1-hour and 3-hours after delivery showed significant shorter values from those obtained during non-pregnancy.

4) PT change (Fig. 3)

The PT values of pregnancy $10 \mathrm{M}$, 1-hour, 3-hours, 4-days, 7-days, 3-weeks, 1-month after delivery and non-pregnant females were $12.6 \pm 0.8,12.9 \pm 1.4,12.9 \pm 1.9,12.6 \pm 0.9,13.3 \pm 0.8,12.7 \pm 0.6$, $12.9 \pm 0.5$, and $(12.1 \pm 0.7)$ seconds, respectively. PT showed no significant differences between the adjoining groups. However, 7-days after delivery PT showed significant higher values compared with non-pregnancy.

\section{5) Fibrinogen concentration change (Fig. 4)}

Fibrinogen concentrations during pregnancy $10 \mathrm{M}$, 1-hour, 3-hours, 4-days, 7-days, 3-weeks, 1-month after delivery and in non-pregnant females were $498.6 \pm 56.0,520.8 \pm 52.4,498.5 \pm 53.5,439.4 \pm 75.3$, $381.6 \pm 61.2,283.6 \pm 67.9,261.3 \pm 43.7$, and $(253.0 \pm 39.0) \mathrm{mg} / \mathrm{dl}$, respectively. Fibrinogen concentration values at 1-hour, 3-hours, and 4-days were within the normal pregnancy range of $10 \mathrm{M}$, with no significant differences between the adjoining groups. The values at 3-weeks and 1-month were within the normal range of non-pregnant females with no significant differences between the two groups. The value at 7-days showed significant differences from at Pregnancy $10 \mathrm{M}$ and non-pregnancy, respectively. 


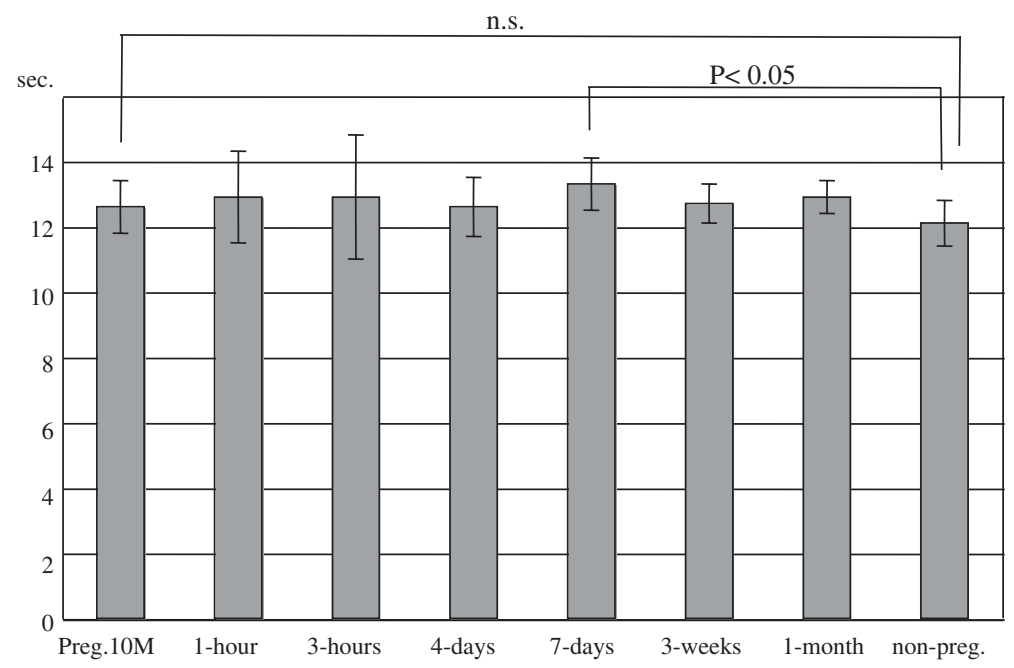

Fig. 3. PT change. No significant differences between the adjoining groups with the level of significance of 5\%.

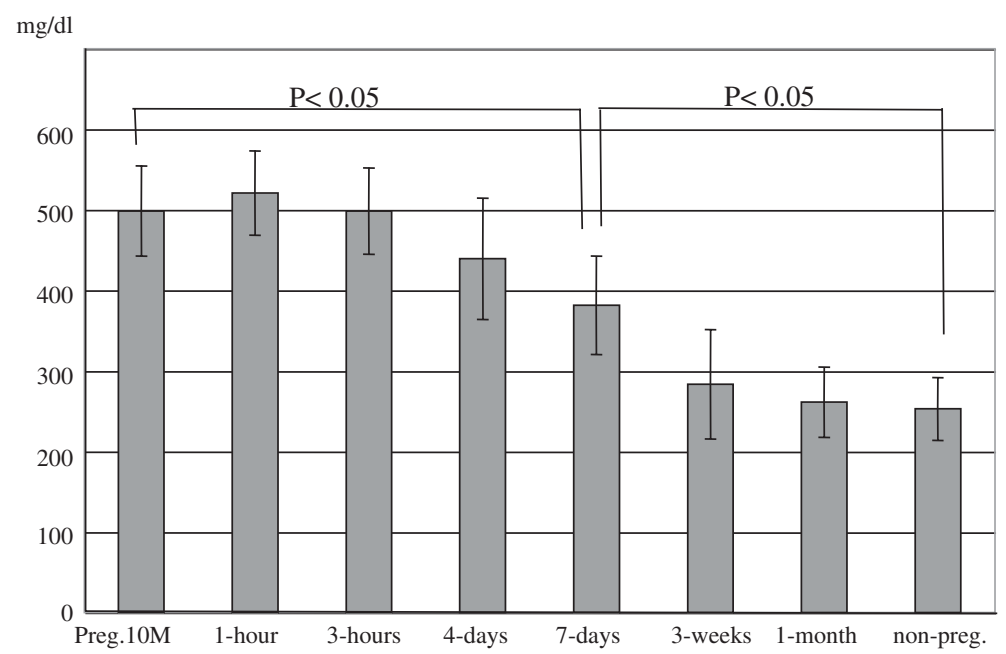

Fig. 4. Fibrinogen concentration. Fibrinogen concentration values at the third trimester, 1-hour, 3-hours, 4-days, 7-days were within normal range of the third trimester of pregnancy, showing no significant differences between the adjoining groups. The values at 3-weeks and 1-month were within normal range of non-pregnant female showing no significant differences between the two groups.

\section{6) Ti change (Fig. 5)}

Ti values of pregnancy $10 \mathrm{M}$, 1-hour, 3-hours, 4-days, 7-days, 3-weeks, 1-month after delivery and in non-pregnant females were $20.4 \pm 2.2,11.7 \pm 1.6,13.2 \pm 3.1,17.2 \pm 2.0,20.2 \pm 1.6,21.4 \pm 4.0$, $24.6 \pm 3.6$, and $(25.0 \pm 3.4)$ minutes, respectively. Ti was the shortest at one hour after delivery, which was almost half of that of pregnancy $10 \mathrm{M}$. 


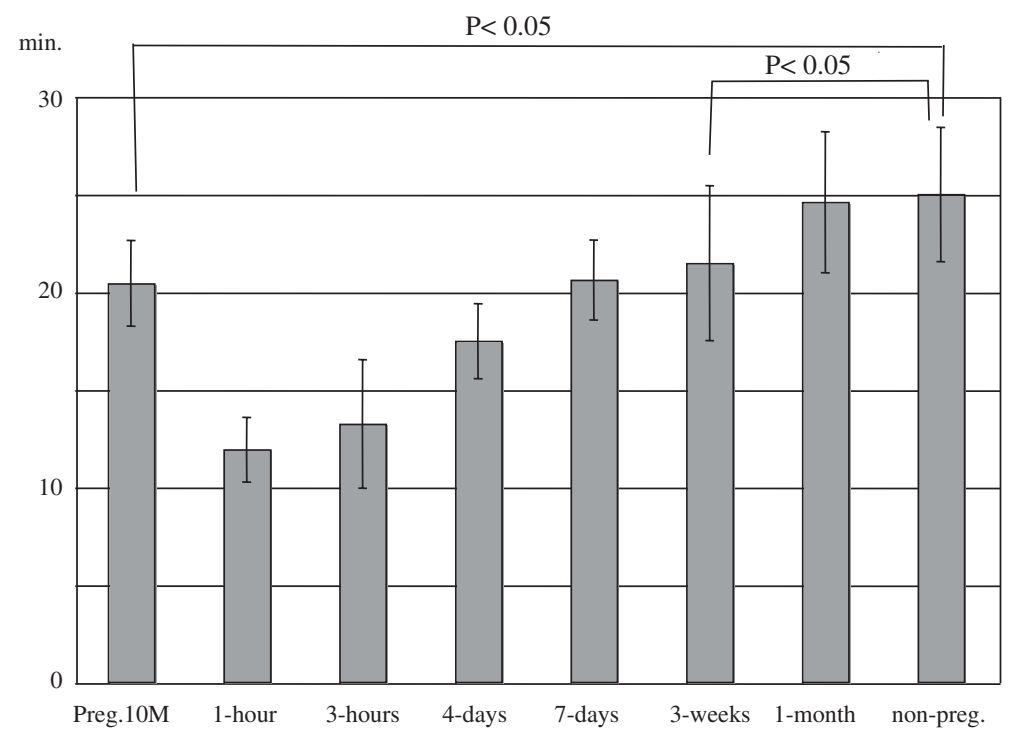

Fig. 5. Ti change. Ti showed the shortest time at 1-hour after delivery, which was almost half of that of the third trimester of pregnancy. 3-hours after delivery showed almost same value of Ti as one hour after delivery. It needs about one week to recover Ti value of the third trimester. Ti of 1-month and non-pregnant showed no significant difference with the level of significance of 5\%.

\section{Discussion}

Obstetric embolism has been increasing for the past 10 years as a cause of perinatal maternal death, more than pre-eclampsia and postpartum hemorrhage [21]. Pulmonary embolism is the most frequent cause of maternal death in the United States [2]. No marked changes have been shown for at least 10 years, although this is possibly related to recognition of pulmonary embolism by obstetricians and improved diagnostic technology. Risk factors of deep venous thrombosis as causes of pulmonary embolism include older age, surgery, trauma, malignancy, thrombophilia, immobilization, varicose veins, obesity, estrogen use and pregnancy, etc. [6]. On the other hand, thromboembolism in pregnancy occurs at a rate of $0.5-3 / 1,000$ $[3,23]$. Earlier studies indicated that the incidence of postnatal thromboembolism was greater than that during pregnancy. However, the incidence of the latter was similar to the former considering the effects of transvaginal delivery and recumbency after cesarean delivery and hospitalization [23]. However, it is unlikely that blood in late pregnancy, which is significantly more hypercoagulable compared with nonpregnant blood, exhibits less of a change in coagulation after delivery associated with bleeding. Therefore, we determined the time of initiation of coagulation from late pregnancy up to one month after delivery and evaluated the tendency of thrombus formation in pregnant and postpartum women.

When measuring APTT, PT, fibrinogen concentrations and platelet concentrations, we did not consider RBC concentrations in this study. It is well known that RBC concentrations significantly influence hemostasis $[11,24,27]$. However, we have already confirmed that females with low RBC concentrations showed virtually the same $\mathrm{Ti}$ as females with normal RBC concentrations at pregnancy 10M [25]. These data suggest that blood in late pregnancy had little effect on the time of initiation of coagulation, even when the concentration of red blood cells is low, i.e., if the subject has anemia. Therefore, it was considered that time of initiation of coagulation of postpartum women might be evaluated without strict grouping of 
the concentration of red blood cells. The time of initiation of coagulation in the blood from postpartum women was determined using a damped oscillation-type rheometer immediately after collection of the blood.

Ti was the shortest at one hour after delivery, which was almost half of that of pregnancy $10 \mathrm{M}$. Three hours post delivery showed almost the same Ti value as at one hour after delivery. Ti was prolonged up to three weeks after delivery, and showed significantly shorter values compared with non-pregnant females. These data might suggest that pregnant women are at increased risk of thrombosis up to three weeks after delivery.

Gerbasi et al. [7] suggested a marked increase in platelet activation immediately after placental delivery and for the first 3 hours post partum, based upon measurement of platelet parameters, i.e., platelet counts, platelet volume, platelet factor 4 and B-thromboglobulin. Based on an increase in fibrinopeptide A and corresponding decreases in fibrinogen and antithrombin III, they suggested an increase in clotting system activity, immediately after placental delivery and for the first 3 hours post partum. Our data that Ti at 1 - and 3-hours after delivery were almost half of that of pregnancy $10 \mathrm{M}$ was similar to the suggestion of Gerbasi et al. Lim et al. [20] reported that the coagulation time was inversely proportional to the concentration of fibrinogen. However, it was impossible for us to predict the dynamic changes of $\mathrm{Ti}$ around delivery based on the values of APTT, PT and fibrinogen concentrations.

Sharma et al. measured the blood coagulation of pregnant women ( $>36$ week gestation) and in women presenting for postpartum tubal ligation 12 24 hours post delivery using TEG, and showed that pregnancy is an hypercoagulable state and that postpartum women remain hypercoagulable throughout the first 24 hours post delivery [26]. Our data support that the risk of hypercoagulability is highest just after the delivery. Venous thromboembolism (VTE) post delivery was seen most at the first day, and $67 \%$ was in the three days post partum [19]. Our data are not consistent with these reports.

Hellgren reported that acute phase reactions in expressed as increased levels of C-reactive protein, fibrinogen, and platelets and antithrombin levels increase during the first week post partum [13]. It requires about one week to recover to a normal Ti value of pregnancy $10 \mathrm{M}$, and up to one month to restore to non-pregnant levels. The Ti of 1-month and in non-pregnant rates also showed no significant difference $(P>0.05)$.

The results of this study suggested that the blood of postpartum women immediately after delivery and 1 and 3 hours after initiated coagulation at about half of the time of pregnant women and that postpartum women had a higher tendency of thrombus formation compared with late pregnant women before delivery. Therefore, it is important to make the blood flow well by moving the lower limbs of postpartum women while in bed within 3 hours after delivery to prevent thrombus formation. The conditions of the significantly earlier initiation of coagulation compared with those in nonpregnant women remained for approximately 3 weeks and it took approximately one month to return to the point where there was no significance between postpartum and nonpregnant women. To be specific, the blood of postpartum women is hypercoagulable up to 3 weeks after delivery and also at home, therefore, it is necessary to consider them to have a tendency of higher thrombus formation and observe, consult, instruct and treat postpartum women with obesity, varicose vein and vulval varices.

\section{References}

[1] Anonymus, Ethical guidelines for publication in Clinical Hemorheology and Microcirculation, Clin Hemorheol Microcirc 44(1) (2010), 1-2. 
[2] R.L. Barbieri and J.T. Repke, Medical disorders during pregnancy, In: Harrison's Principles of Internal Medicine, D.L. Kasper, E. Braunwald, A.S. Fauci, S.L. Huser, D.L. Longo, J.L. Jameson Eds., 16th Edn., McGraw-Hill Companies Inc., New York, 2005, 32-38.

[3] L.A. Barbour and J. Pickard, Controversies in thromboembolic disease during pregnancy: A critical review, Obstet Gynecol 86 (1995), 621-633.

[4] O.K. Baskurt, M. Boynard, G.C. Cokelet, P. Connes, B.M. Cooke, S. Forconi, F. Liao, M.R. Hardeman, F. Jung, H.J. Meiselman, G. Nash, N. Nemeth, B. Neu, B. Sandhagen, S. Shin, G. Thurston and J.L. Wautier, International expert panel for standardization of hemorheological methods, New guidelines for hemorheological laboratory techniques, Clin Hemorheol Microcirc 42(2) (2009), 75-97.

[5] P. Clark, Changes of hemostasis variables during pregnancy, Semin Vascul Med 3 (2003), 13-24.

[6] C.W. Francis and K.L. Kaplan, Venous and arterial thrombosis, In: Clinical Hematology, N.S. Young, S.L. Gerson and K.A. High Eds., Elsevier Inc. Pennsylvania, 2006, 1089-1105.

[7] F.R. Gerbasi, S. Bottoms, A. Farag and E.F. Mammen, Changes in hemostasis activity during delivery and the immediate postpartum period, Am J Obstet Gynecol 162 (1990), 1158-1163.

[8] J.S. Ginsberg, P. Brill-Edwards, R.F. Burrows, et al., Venous thrombosis during pregnancy: leg and trimester of presentation, Thromb Haemost 67 (1992), 519-520.

[9] I.A. Greer, Prevention of venous thromboembolism in pregnancy, Best Pract Res Clin Haematol 16 (2003), 261-278.

[10] Haematology ICfSi. Guidelines for measurement of blood viscosity and erythrocyte deformability. (Expert panel on blood rheology), Clincal Hemorheology 6 (1986), 439-453.

[11] L. Heilmann, S. Gelhold, G.-F. von Temelhoff and K. Pollow, The role of intravenous volume expansion in moderate pre-eclampsia, Clin Hemorheol Microcirc 25 (2001), 83-89.

[12] M. Hellgren, Hemostasis during normal pregnancy and puerperium, Semin Thromb Hemost 29 (2003), 125-130.

[13] M. Hellgren and M. Blombäck, Studies on blood coagulation and fibrinolysis in Pregnancy, during delivery and in the puerperium, Gynecol Obstet Invest 12 (1981), 141-154.

[14] H. Iwata, H. Kasuga, M. Nakayama, M. Kaibara and H. Sasabe, Changes in rheological property and fibrin network during coagulation and fibrinolysis, Rept Progr Polym Phy Jpn 40 (1997), 585-588.

[15] M. Kaibara and M. Date, A new rheological method to measure fluidity change of blood during coagulation; application to in vitro evaluation of anticoagulability of artificial materials, Biorheology 22 (1985), 197-208.

[16] M. Kaibara, Y. Kawamoto, S. Yanagida and S. Kawakami, In vitro evaluation of antithrombogenicity of hybridtype vascular vessel models based on analysis of the mechanism of blood coagulation, Biomaterials 16 (1995), $1229-1234$.

[17] S. Kawakami, M. Kaibara, Y. Kawamoto and K. Yamanaka, Rheological approach to the analysis of blood coagulation in endothelial cell-coated tubes: activation of the intrinsic reaction on the erythrocyte surface, Biorheology 32 (1995), 521-536.

[18] Y. Kawamoto and M. Kaibara, Procoagulant activity of collagen, Effect of difference in type and structure of collagen, Biochem Biophys Acta 1035 (1990), 361-368.

[19] T. Kobayashi, M. Nakabayashi, M. Ishikawa, et al., Final reports of deep vein thrombosis/pulmonary thromboembolism between 1991 and 2000 in Obstetrics and Gynecology, Jpn J Obstet Gynecol Neonatal Hematol 14 (2005), $1-24$.

[20] H. Lim, J. Nam, S. Xue and S. Shin, Measurement of blood coagulation with considering RBC aggregation through a microchip-based light transmission aggregometer, Clin Hemorheol Microcirc 47(3) (2011), 211-218.

[21] Mothers' and Children's Health and Welfare Association, Maternal and child health statistics of Japan, Tokyo, Japan, 2010, pp. 79-80.

[22] F.R. Rosendaal, Risk factors for venous thrombotic disease, Thromb Haemost 82 (1999), 610-619.

[23] S. Rutherford, M. Montoro, W. McGehee and T. Strong, Thromboembolic desease associated with pregnancy: An 11-year review, Am J Obstet Gynecol 164 (1991), 286.

[24] T. Sagesaka, Influence of red blood cell concentration on the initiation time of blood coagulation: Risk of thrombus formation by hemoconcentration, Clin Hemorheol Microcirc 31 (2004), 243-249.

[25] T. Sagesaka, H. Juen and M. Hayashi, Influence of red blood cell concentration on the initiation time of blood coagulation: Risk of thrombus formation in pregnant females with anemia, Clin Hemorheol Microcirc 36 (2007), $155-161$.

[26] S.K. Sharma, J. Philip and J. Wiley, Thromboelastographic changes in healthy parturients and postpartum women, Anesth Analg 85 (1997), 94-98. 
[27] V. Turitto and H. Weiss, Red, blood cells: Their dual role in thrombus formation, Science 207 (1980), 541-543.

[28] G.F. von Tempelhoff, F. Nieman, L. Heilmann and G. Hommel, Association between blood rheology, thrombosis and cancer survival in patients with gynecologic malignancy, Clin Hemorheol Microcirc 22(2) (2000), 107-130. 